\title{
Metabolic Effects of Foenugreek, Nigella and Termis: Review Article
}

\author{
Waleed S. Mohamed ${ }^{*} 1$, a \\ ${ }^{1}$ Internal Medicine Department, College of Medicine, Taif University, \\ KSA and Internal Medicine Department, Tanta Faculty of Medicine, Tanta University, Egypt \\ a email: wsmohamed1@yahoo.com
}

\begin{abstract}
Keywords: Alloxan - Antidiabetic plants - Diabetes - Pancreas.
\end{abstract}
\begin{abstract}
Diabetes is a chronic metabolic disease, which affects large number of population all over the world. Diabetes is associated with many complications as dyslipidemia, coronary heart diseases and non alcoholic fatty liver diseases. More than 400 traditional medicinal plants have been recorded for helping in controlling such disease. This review discusses the effects of most common plants (Foenugreek, Nigella and Termis) used in Saudi Arabia and some other Arab countries as antidiabetic agents. The usage of the mixture or each plant alone corrected the blood glucose level and increase insulin secretion with definite increase in the number and diameter of beta pancreatic cells in the diabetic rats. Also, improve the associated dyslipidemia, and ameliorated most of all damage effects of Alloxan on hematological parameters present in diabetic rats. So, it can control most of the metabolic risk factors of coronary artery disease (CAD) in diabetic. Finally, it appeared to be a useful agent in improving fatty changes in the liver texture associated with DM. It can be concluded that, water extract of the mixture of Foenugreek, Nigella and Termis is the most powerful in amelioration hyperglycemia, dyslipidemia and most of all damage effects of Alloxan on the liver and hematological parameters.
\end{abstract}

\section{Introduction}

Traditional remedies (Folk Medicine), which are often free from side effects are still in use as medicines by a majority of cultures worldwide, and may therefore; present new avenues in the search for alternative drugs. The term of medicinal plants include a various types of plants used in herbalism and some of these plants have a medicinal activities. These medicinal plants or their derivatives consider as a rich resources of ingredients, which can be used in drug development and synthesis. Nowadays, the term "Alternative Medicine" became very common in western culture, it focus on the idea of using the plants for medicinal purpose. Medicinal plants have a promising future because there are about half million plants around the world, and most of them their medical activities have not investigate yet, therefore it is a very important to clarify their role in the treatment of present diseases, and how they can be used to produce or synthesis more effective drugs [1]. Foenugreek, Nigella and Termis are examples of folk medicine which are used widely in Arabic area as herbal medicine for many diseases especially diabetes mellitus (DM) and many published reports demonstrate their medicinal effects.

\section{Anti-diabetic effect}

DM is possibly the world's fastest growing metabolic disease, so there is a great need for more appropriate therapies Many plants have been investigated for their beneficial use in treating DM [2]. Nigella sativa oil lowered blood glucose concentration in the diabetic rats and improved altered levels of lipid peroxidation products. Abdel Moneim et al. [3] reported that the hypoglycemic effect of Nigella sativa may be attributed to an increase in the islet numbers and to its effect on the timecourse of glucose resorption from the intestine. Its hypoglycemic effect may be mediated by extra pancreatic actions rather than by stimulated insulin release. Also, its antioxidant effects may be useful in controlling the diabetic complications [4, 5]. Oral administration of ethanolic extract of Nigella sativa seeds to streptozotocin induced diabetic rats for 30 days reduced the elevated levels of blood glucose and improved altered levels of lipid peroxidation products. Fenugreek (Trigonella 
foenum-gracaeum) may increase plasma insulin level in vivo [6]. Various hypotheses about the mechanism of the hypoglycemic activity of Foenugreek have been postulated, including delayed gastric emptying and an agonist effect on insulin receptors [7]. The major free amino acid 4hydroxyisoleucine constituent of Foenugreek stimulates insulin secretion from perfuse pancreas in vitro [8].

Termis seeds (Lupine) are a medicinal plant with potential antihyperglycemic activity present in extracts of the whole seed. In white mice, extracts of seeds of the white Termis were associated with increased tolerance to an oral glucose [9]. Mostafa et al., [10] found that, induction of diabetes by alloxan in rats cause a significant decrease in beta cells number and increase in beta cells diameter as well as nuclear diameters. Water extract of Foenugreek, Nigella and Termis appeared to be useful agents in reducing the hyperglycemia by increasing insulin level and regenerating beta cells of the pancreas (increase in beta cell number and a decrease in their diameters as well a nuclear diameter) and may block the diabetogenic action of Alloxan and restore insulin production. However, a mixture of these plants proved to be more effective than each of them without added side effects. Also, Augusti and Sheela [11] mentioned that some plants exert their effect on beta cells through both protection of the already present beta cells due to their antioxidant effect and through stimulation of the beta cells to release insulin. Marles et al. [12] suggested that, the hypoglycemic effect of some medicinal plants could be attributed to factors other than stimulation of insulin release only, e.g. their effect on the number and /or affinity of insulin receptors on target cells and the post-receptors of these cells.

\section{Anti-dyslipidemic effect}

Patients with type 2 DM (T2DM) have a higher incidence of dyslipidemia, and obesity, which contribute to their high risk of CHD [13]. Disturbance of lipid metabolism appears to be an early event in the development of T2DM, potentially preceding the disease by several years [14]. The dyslipidemia spectrum in DM can include all the various types of dyslipidemia identified in the general population; however, the characteristic features are a high plasma triglyceride (TG) concentration, increased concentration of small density lipoprotein (LDL) particles and low high density lipoprotein (HDL) concentration which are attributed mostly to insulin resistance (IR) and insulin deficiency [15]. The main cause of diabetic dyslipidemia is the increased free fatty acid release from IR fat cells into the liver in the presence of adequate glycogen stores promotes TG production, which in turn stimulates the secretion very low density lipoprotein cholesterol (VLDL) [16]. The increased number of VLDL cholesterol particles and increased plasma TG levels decrease the level of HDL and increase the concentration of small, dense LDL particles via several processes: VLDL transported TG is exchanged for HDL transported cholesteryl ester through the action of the cholesteryl ester transfer protein (CETP), which results in increased both atherogenic cholesterol-rich VLDL remnant particles and TG rich cholesterol depleted HDL particles [18].

The literature survey revealed that Nigella has been shown to produce multi-systemic beneficial actions including hypocholestermic, hypoglycemic and antioxidant effects $[5,18]$. The presence of proteins and fiber (galactomannan) in Foenugreek seeds might exert a lipid lowering effect and may form a viscous gel in the intestine and inhibit glucose and lipid absorption [19]. Some studies observed a beneficial influence of Lupin protein on blood cholesterol concentrations and also partially in blood pressure [20]. Waleed et al., [21] found that the aqueous extract of the Fenugreek and a mixture of Nigella, Foenugreek and Termis are the most powerful in amelioration of lipid abnormalities present in diabetic rats as it significantly decreased the levels of total lipids, cholesterol, TG and LDL with non-significant increase in HDL level either through control of DM and direct action on associated dyslipidemia without added side effects. This may attributed to their stimulation to the most aspects of carbohydrate metabolism, including rapid uptake of glucose by the cells, enhanced gluconeogenesis, increased rate of absorption from the gastrointestinal tract and even increased insulin secretion with its resultant secondary effects on carbohydrate metabolism [22]. Zahida et al. [23] found that Nigella decreased cholesterol 14.58\%, LDL 23\%, triglycerides $15.16 \%$ and VLDL $15.16 \%$, whereas HDL increased $3.18 \%$, significantly after six months of 
treatment and concluded that Nigella has ability to reduce the lipid profile, which is a major risk factor for CHD in cardiac patients.

Ghazi Mahmood et al. [24] stated that, Nigella is more effective than Gemfibrozil in hyperlipidemia and it can be used as an add on drug therapy in patients of metabolic syndrome with poor glycemic control. The most important action of Nigella that may be responsible for its beneficial effect in metabolic syndrome is its insulin sensitizing action. Similarly the effects of Nigella seeds on the blood levels of cholesterol, triglycerides, HDL and LDL in white albino rats were studied by Ali et al., [25] a total of 200 rats, 150 experimental and 50 controlled were included in the study. Six doses of Nigella sativa were used (50,100, 200, 300, 400 and $500 \mathrm{mg} / \mathrm{day} / 200 \mathrm{~g}$ rat). The dose was given for five durations: 1, 4, 7, 10, and 14 days. Generally, all doses of Nigella sativa produced significant reduction in the blood level of all studied parameters. There was no linear dose or time dependent effect of Nigella sativa on these parameters. The effect of Nigella sativa started after 4 days and continued some swings, for the rest of the duration. The effective dose of Nigella sativa seemed to lie between $100-400 \mathrm{mg} / \mathrm{kg}$. The carbonyl fraction of the seed extract has got excellent choleretic activity which may affect the body total lipid concentration and help prevent atherosclerosis [23].

Sirtori et al. [26] also showed that Lupin protein isolates were able to reduce plasma total, VLDL and LDL cholesterol concentrations in rats. This effect was associated with the stimulation of LDL receptors by a well-defined protein component of the Lupin seeds, as demonstrated by in vitro studies. Yoshie-Stark and Wa"sche, [27] also showed that the application of Lupin-isolated protein had the capacity to bind bile acids to nearly the same extent as cholestyramine. Similarly, Martins et al., [28] found a hypocholesterolemic effect of Termis and stated that this effect was mainly the consequence of a marked decrease in the intestinal absorption of cholesterol probably modulated by bile acid reabsorption and a higher content of dietary phytosterols. Bähr et al., [29] found that a modest amount comprising $25.0 \mathrm{~g} / \mathrm{d}$ of additionally consumed Termis is capable of lowering total and LDL cholesterol concentrations as well as the LDL: HDL cholesterol ratio from baseline to week 4, primarily in subjects with higher hypercholesterolemia. Proteins, galactomannan, and polyphenols from Foenugreek seeds have been reported to regulate dyslipidemia in obese and diabetic rodents. The presence of these phytoconstituents effectively inhibits fat accumulation and ameliorates dyslipidemia in obese rats, which is due to improvement in glucose and lipid metabolism, increased insulin sensitivity; improve antioxidant defense, and downregulation of lipogenic enzymes [30].

\section{Hematological effect}

Alloxan significantly decrease red blood cells (RBCs) number, hematocrit (HCT) and hemoglobin $(\mathrm{Hb})$ levels in the diabetic induced animals with no effect on WBCs number. The water extract of the mixture of Foenugreek, Nigella and Termis statistically increased RBCs, HCT, and $\mathrm{Hb}$ in all the treated rats. Several investigators attributed this anemia to the increase in lipid peroxidation of the erythrocyte cell membrane by the destructive effect of alloxan [24].

\section{Cardiometabolic effect}

CHD is a major complication of diabetes and the leading cause of eath among diabetic people. People with T2DM also have high rates of high blood pressure, dyslipidemia, and obesity, which contribute to their high rates of CHD [31]. The United Kingdom Prospective Diabetes Study, [32] and other clinical trials have demonstrated the following benefits of optimal control of the of diabetes: Intensive glucose control reduces the risk of any CHD event by 42 percent and the risk of heart attack, stroke, or death from cardiovascular diseases (CVD) by 57 percent. In general, every percentage point drop in hemoglobin $A 1_{C}\left(A 1_{C}\right)$ results reduces the risk of diabetes, kidney, eye, and nerve disease by 40 percent. Increased level of blood cholesterol, LDL, TG, smoking and raised blood pressure is established as major risk factors for coronary heart disease [33]. Improved control of blood lipids (cholesterol, HDL, LDL, and TG) can reduce CHD complications by 20 to 
50 percent [34]. Our previous work [10] revealed that, the water extract of the mixture of these plants may have a cardiometabolic protective effect by controlling most of metabolic risk factors of CHD in diabetic rats through reducing the hyperglycemia, amelioration of all damage effects of Alloxan on hematological parameters, and correction lipid profile abnormalities.

\section{Effect on Nonalcoholic fatty liver disease (NAFLD)}

NAFLD is an increasingly recognized condition that may progress to end-stage liver disease, affecting approximately $30 \%$ of Western populations [35]. DM per se may generate NAFLD, in association with obesity and dyslipidemia [36]. NAFLD is now considered as the hepatic manifestation of the metabolic syndrome, and it is present in approximately $80 \%$ of cases of T2 DM [37]. It was found that, in patients with T2DM with or without obesity, up to $30 \%$ have fat with inflammation, 25\% had associated fibrosis, and 1-8\% had cirrhosis [38]. In NAFLD, DM constitutes a risk factor for non alcoholic steatohepatitis (NASH) and advanced progressive liver disease [39]. Martin et al. [40] reported that the development of NAFLD comes from an imbalance between the influx and production of fatty acids and the use of fatty acids for oxidation or secretion as VLDL triglycerides TGs. Mechanisms leading to lipid accumulation are not completely understood, but it could potentially result from insulin resistance [41] and decreased disposal of fatty acids from impaired mitochondrial $ß$-oxidation or deficient production of very low-density lipoprotein (VLDL) [39]. The proinflammatory and profibrogenic properties of lipid peroxidation end products such as serum malondialdehyde (MDA) potentially account for all of the typical histological features observed in this disorder [42].

Some researchers found that excess glycogen accumulation in the liver is seen in $80 \%$ of diabetic patients as glycogen synthesis is impaired in the liver due to defective activation of glycogen synthase and it was postulated that long-standing insulin deficiency may actually facilitate synthase activity [43]. The steatosis may be microvesicular or macrovesicular, and it may progress to fibrosis and cirrhosis [38]. From our previous work, we found fatty changes in alloxan induced DM with a significant increase in AST, ALT, and LDH levels and a significant decrease in the serum protein level. In addition, in diabetic liver periportal fibrosis, vacuolated cytoplasm and cellular infiltration were observed. The traditional antidiabetic plants might provide a useful source of new oral hypoglycaemic compounds, and they have a good effect on associated liver diseases. This may be attributed to their stimulation of most aspects of carbohydrate metabolism, including the rapid uptake of glucose by the cells, enhanced gluconeogenesis, increased rate of absorption from the gastrointestinal tract, regenerating pancreatic b-cell, and even increased insulin secretion [44].

Oral administration of an ethanolic extract of N. sativa seeds to streptozotocin-induced diabetic rats for 30 days reduced the elevated levels of blood glucose and improved the altered levels of lipid peroxidation products; in addition, because of its antioxidant effects, its administration may be useful in controlling diabetic complications [38,41]. Fenugreek may increase the plasma insulin level in vivo [39], delayed gastric emptying and an agonist effect on insulin receptors [34]. Termis seeds potential value in the management of diabetes with antihyperglycaemic activity, and was associated with increased tolerance to oral glucose [9]. A significant decrease in AST, ALT, and LDH levels occurred more marked in the mixture-treated group. Furthermore, the TP levels were significantly raised in all treated groups. In a recent point prevalence study in $>9000 \mathrm{~T} 2 \mathrm{DM}$ patients in eight Italian centers, the overall prevalence of raised ALT and AST was $16 \%$ and $8.8 \%$, respectively [45]. A histological study of diabetic rat liver sections after a 30-day treatment demonstrated histological improvement of the liver texture, especially in a diabetic mixture-treated rat than the liver of a diabetic N. sativa-treated rat and the liver of a diabetic fenugreek-treated rat with minimal effects observed in the termis-treated rat [44]. In conclusion an aqueous extract of the seed mixture of the studied plants appeared to be useful agents in reducing fatty changes in the liver texture. 


\section{Conclusion}

The water extract of the mixture of Foenugreek, Nigella and Termis is the most powerful in amelioration hyperglycemia, dyslipidemia and most of all damage effects of Alloxan on the liver and hematological parameters.

\section{Recommendations}

It is advised to use the plant mixture as an antidiabetic agent rather than the use of each plant separately. More studies on these plants are advised be done with different doses and for different periods before recommending their use on a wide scale.

\section{References}

[1] Rasool Hassan. Medicinal Plants (Importance and Uses). Pharmaceut Anal Acta. 3 (2012) $1000 \mathrm{e} 139$.

[2] Maiti R, Jana D, Das U, Ghosh D. Antidiabetic effect of aqueous extract of seed of tamarindus indica in streptozotocin induced diabetic rats. J Ethnopharmacol 92 (2004) 85-91.

[3] Abdel-Moneim A, El-Feki M and Salah E. Effect of Nigella Sativa, fish oil and gliclazide on Alloxan-diabetic rats. Biochemical and histopathological studies. J Egypt Ger Sc Zool. 23 (1999) 237-265.

[4] El-Dakhakhny M, Mady N, Lembert N and Ammon HP. The hypoglycemic effect of Nigella sativa oil is mediated by extrapancreatic actions. Planta Med. 68 (2002) 465-466.

[5] Kaleem M, Kirmani D, Asif M, Ahmed Q, Bano B. Biochemical effects of Nigella sativa L seeds in diabetic rats. Indian J Exp Biol 44 (2006) 745-748.

[6] Kavishankar G, Lakshmidevi N, Mahadeva M, Prakash H. Diabetes and medicinal plants-A review. Int J Pharm Biomed Sci. 2 (2011) 65-80.

[7] Wadkar K, Magdum C, Patil S, Naikwade N. Antidiabetic potential and Indian medicinal plants. J Herbal Med and Toxicol. 2 (2008) 45-50.

[8] Al-Habbori M, Raman A. Antidiabetic and hypocholesterolemic effects of fenugreek. Phytotherapy Research. 12 (1998) 233-242.

[9] Knecht K, Nguyen H, Auker A, Kinder D. Effects of extracts of lupine seed on blood glucose levels in glucose resistant mice: antihyperglycemic effects of Lupinus albus (white lupine, Egypt) and Lupinus caudatus. J. Herb Pharmacother. 6 (2006) 89-104.

[10] Mostafa AM, Serwah AH, Mohamed WS, Mohamed KM. Effects of some antidiabetic medicinal plants on pancreas and liver of diabetic Albino rats. Egyptian Journal of Hospital Medicine. 50 (2013) 156-168.

[11] Augusti K. and Sheela G. Theory and Practice of Histological Techniques. $4^{\text {th }}$ edition. Churchill Living. Edinburgh and London. 1996; p.123.

[12] Meral I, Yener Z, Kahraman T, Mert N. Effect of Nigella sativa on glucose concentration, lipid peroxidation, anti-oxidant defense system and liver damage in experimentally induced diabetic rabbits. J. Vet. Med. A. Physiol. Pathol. Clin. Med. 48 (2001) 593-599.

[13] Krishnaswami Vijayaraghavan. Treatment of dyslipidemia in patients with type 2 diabetes. Lipids in Health and Disease 2010; 9: 144-156.

[14] Saydah SH, Fradkin J, Cowie CC. Poor control of risk factors for vascular disease among adults with previously diagnosed diabetes. JAMA 2004; 291: 335-342.

[15] Chahil TJ, Ginsberg HN. Diabetic dyslipidemia. Endocrinol Metab Clin North Am. 35 (2006) 491-510.

[16] Adiels M et al. Acute suppression of VLDL1 secretion rate of insulin is associated with hepatic fat content and insulin resistance. Diabetologia. 50 (2007) 2356-2365.

[17] Mooradian AD et al. Obesity-related changes in high density lipoprotein metabolism. Obesity 16 (2008) 1152-1160. 
[18] Marzouk M, Soliman A, Omar T. "Hypoglycemic and antioxidative effects of fenugreek and termis seeds powder in streptozotocin-diabetic rats," European Review for Medical and Pharmacological Sciences. 17 (2013) 559-565.

[19] Hamden K, Jaouadi B, Carreau S, Bejar S, Elfeki A. "Inhibitory effect of fenugreek galactomannan on digestive enzymes related to diabetes, hyperlipidemia, and liver-kidney dysfunctions. Biotechnology and Bioprocess Engineering. 15 (2010) 407-413.

[20] Naruszewicz M, Nowicka G, Klosiewicz-Latoszek L, Arnoldi A, Sirtori C. Effect of Lupin protein (Lupinus Albus) on cardiovascular risk factors in smokers with mild hypercholesterolemia [abstract]. Circulation 114 (2006) 874.

[21] Mohamed WS, Mostafa AM, Mohamed KM, Serwah AH. The effects of Fenugreek, Nigella and Termis seeds on lipid profile in diabetic albino rats. Annals of Biological Sciences 3 (2015) 1-9.

[22] Duke J. Handbook of medicinal Herbs. 2nd ed. United States of America 2002, Pp: 15-51.

[23] Zahida T, Zeshan S, Nisar A, Mushtaq H. The Effects of Nigella sativa (Kalonji) on Lipid Profile in Patients with Stable Coronary Artery Disease in Multan, Pakistan. Pakistan Journal of Nutrition 10 (2011) 162-167.

[24] Ghazi Mahmood, Aruna Bashir, Shah Murad, Javed Asghar, Shaheena Asif, Abdul Majeed Ch, Muhammad Aslam. Comparison of lipid lowering effects of Nigella Sativa and Gemfibrozil. International Journal of Pharmaceutical Research \& Development 3 (2012) 6-10.

[25] Ali B, Blunden G. Pharmacological and toxicological properties of Nigella sativa. Phytother Res. 17 (2003) 299-305.

[26] Sirtori C, Lovati M, C Manzoni, Castiglioni S, Duranti M, Magni C, Morandi S, D’Agostina A, Arnoldi A. Proteins of white lupin seed, a naturally isoflavonepoor legume, reduce cholesterolemia in rats and increase LDL receptor activity in HepG2 cells. J Nutr. 134 (2004) 18-23.

[27] Yoshie-Stark Y, Wa"sche A. In vitro binding of bile acids by lupin protein isolates and their hydrolysates. Food Chem. 88 (2004) 179-184.

[28] Martins J, Riottot M, de Abreu M, Lanca M, Viegas-Crespo A, Almeida J, Freire J, Bento O. Dietary raw peas (Pisum sativum L.) Reduce plasma total and LDL cholesterol and hepatic esterified cholesterol in intact and ileorectal anastomoses pigs fed cholesterol-rich diets. J Nutr. 134 (2004) 3305-3312.

[29] Bähr M, Fechner A, Krämer J, Kiehntopf M Jahreis G. Lupin protein positively affects plasma LDL cholesterol and LDL: HDL cholesterol ratio in hypercholesterolemic adults after four weeks of supplementation: a randomized, controlled crossover study. Nutrition Journal 12 (2013) 107.

[30] Kumar P, Bhandari U, and Jamadagni S. Fenugreek Seed Extract Inhibit Fat Accumulation and Ameliorates Dyslipidemia in High Fat Diet-Induced Obese Rats. BioMed Research International ( 2014) 11.

[31] Adler A, Stratton I, Neil H, et al. Association of systolic blood pressure with macrovascular and microvascular complications of type 2 diabetes (UKPDS 36): prospective observational study. BMJ 321 (2000) 412-419.

[32] Paromita K, Ian P, and Richard D. The UK Prospective Diabetes Study (UKPDS): clinical and therapeutic implications for type 2 diabetes. Br J Clin Pharmacol. 48 (1999) 643-648.

[33] Helal E, Hasan M, Mustafa A and Al-Kamel A. Effect of Aloe vera extract on some physiological parameters in diabetic albino rats. The Egyptian Journal of Hospital Medicine 12 (2003) 53-61.

[34] Hongxiang H and Vay Liang W. Hypoglycemic herbs and their action mechanisms. Chin Med. 4 (2009) 11-14.

[35] Schreuder T, Verwer B, Nieuwkerk C, Mulder C. Nonalcoholic fatty liver disease. World J Gastroenterol. 14 (2008) 2474-2486.

[36] Takuma Y, Nouso K. Nonalcoholic steatohepatitis-associated hepatocellular carcinoma: our case series and literature review. World J Gastroenterol. 16 (2010) 1436-1441. 
[37] Farrell G, Larter C. Nonalcoholic fatty liver disease: from steatosis to cirrhosis. Hepatology 43 (2006) S99e112.

[38] El-Serag H, Tran T, Everhart J. Diabetes increases the risk of chronic liver disease and hepatocellular carcinoma. Gastroenterology 126 (2004) 460-468.

[39] Edmison J, McCullough AJ. Pathogenesis of non-alcoholic steatohepatitis: human data. Clin Liver Dis. 11(2007) 75-104.

[40] Martin A, Marja-Riitta T, Jan B. Fatty liver, insulin resistance, and dyslipidemia. Curr Diabetes Rep. 8 (2008) 60-64.

[41] Angulo P. Nonalcoholic fatty liver disease. N Engl J Med. 346 (2002) 1221-1231.

[42] Alba L, Lindor K. Review article: non-alcoholic fatty liver disease. Aliment Pharmacol Ther. 17 (2003) 977-986.

[43] Levinthal G, Tavill A. Liver disease and diabetes mellitus. Clinical diabetes 1999; 17(2): 73-7.

[44] Mohamed W, Mostafa A, Mohamed K, Serwah A. "Effects of fenugreek, Nigella, and Termis seeds in nonalcoholic fatty liver in obese diabetic albino rats." Arab Journal of Gastroenterology 16 (2015) 1-9.

[45] Mofrad P, Contos M, Haque M, et al. Clinical and histologic spectrum of nonalcoholic fatty liver disease associated with normal ALT values. Hepatology 37 (2003)1286-1292. 